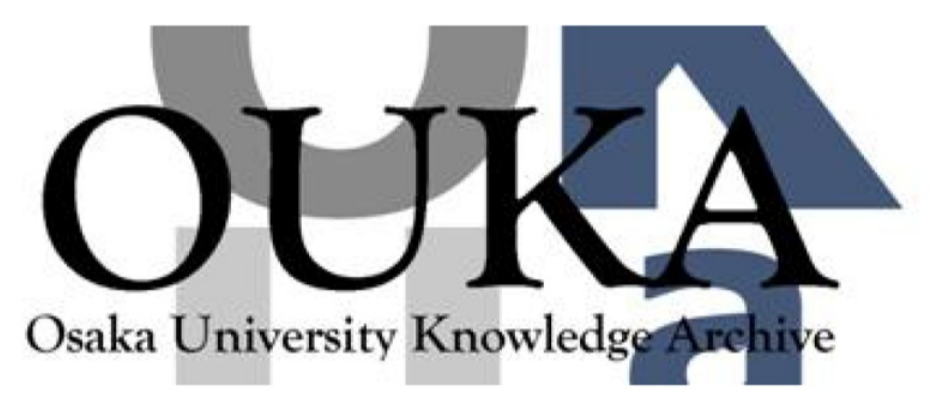

\begin{tabular}{|c|l|}
\hline Title & $\begin{array}{l}\text { Fast recovery of elastic stiffness in Ag thin } \\
\text { film studied by resonant-ultrasound } \\
\text { spectroscopy }\end{array}$ \\
\hline Author(s) & $\begin{array}{l}\text { Nakamura, Nobutomo; Nakashima, Takeo; Ogi, } \\
\text { Hirotsugu et al. }\end{array}$ \\
\hline Citation & $\begin{array}{l}\text { Japanese Journal of Applied Physics. 48 } \\
\text { p.07GA02 }\end{array}$ \\
\hline Issue Date & $2009-07-21$ \\
\hline oaire:version & AM \\
\hline URL & https://hdl.handle. net/11094/84480 \\
\hline rights & \\
\hline Note & \\
\hline
\end{tabular}

Osaka University Knowledge Archive : OUKA

https://ir. Library. osaka-u. ac. jp/

Osaka University 


\section{Fast Recovery of Elastic Stiffness in Ag Thin Film Studied by Resonant-Ultrasound Spectroscopy}

Nobutomo Nakamura*, Takeo Nakashima, Hirotsugu Ogi, Masahiko HiraO, and Masayoshi NishiYama $^{1}$

Graduate School of Engineering Science, Osaka University, 1-3 Machikaneyama, Toyonaka, Osaka 560-8531, Japan

${ }^{1}$ Renovation Center of Instruments for Science Education and Technology, Osaka University, 1-3 Machikaneyama, Toyonaka, Osaka 560-0043, Japan

We studied the recovery of the elastic constant of sputtered Ag films on monocrystal Si substrates by monitoring the resonance frequency of film/substrate specimens throughout the deposition process using resonant-ultrasound spectroscopy. A Ag film is deposited on a (001) Si substrate, which is located on the tripod transducer set in the sputtering chamber. The free-vibration resonance frequency of the $\mathrm{Ag} / \mathrm{Si}$ specimen is measured before, during, and after the magnetron sputtering deposition, inducing the evolution of the elastic constants of the deposited film. Recovery of the elastic constants of the Ag film is completed within $40 \mathrm{~min}$. The recovery behavior of the elastic constants is similar to that of residual stress; the recovery rate is comparable to that for residual stress.

*E-mail address: nobutomo@me.es.osaka-u.ac.jp 


\section{Introduction}

In polycrystalline thin films, the binding strength at grain boundaries governs their macroscopic elastic property. For example, in polycrystalline diamond thin films, incohesive bonding at grain boundaries softens the film, and orderly oriented incohesive bonding induces elastic anisotropy between the in-plane and out-of-plane directions. ${ }^{1,2}$ In nanocrystalline diamond thin films, as grain size decreases, the diagonal components of the elastic matrix decrease and the off-diagonal components increase because of $s p^{2}$ bonding at grain boundaries. ${ }^{3,4}$ For the actual use of thin films, the weak bonding at grain boundaries has to be improved, because it also affects the reliability and functions such as magnetic, electric and optical properties. Therefore, a study of the relationship between the mechanical property and grain boundaries has been an important issue.

Recovery in thin films is one of the phenomena that originate from the change in the binding conditions at grain boundaries after deposition. In bulk materials, the recovery occurs when plastic deformation is generated. However, in thin films, the recovery occurs without applying external forces just after the deposition. Then, the evolution of intrinsic stress occurs with the recovery and has been studied using curvature measurements. ${ }^{5-9}$ In previous studies, compressive stress during deposition changes into tensile stress after deposition. This has been explained by the change in the binding conditions at grain boundaries. ${ }^{10}$

Considering that the elastic constants of thin films are sensitive to the binding strength at grain boundaries, an accurate measurement of the change in the elastic constants allows us to evaluate the recovery in real time. In this study, we study the recovery of elastic constants of silver thin films using resonant-ultrasound spectroscopy. ${ }^{11}$ The recovery rate for the elastic constants is compared with the reported recovery rate for residual stress.

\section{Experimental Procedure}

Recovery of the elastic constants of thin films is detected by measuring the free-vibration resonance frequency of a film/substrate layered specimen. The resonance frequency of the free vibration of a substrate depends on mass density, dimensions, and the elastic constants $C_{i j} .{ }^{12,13}$ When a film is deposited on a substrate, the resonance frequency changes depending on mass density, thickness, and elastic constants of a thin film. By measuring frequency shifts, the elastic constants of a thin film are separately measurable. ${ }^{2,14,15}$ This is called resonantultrasound spectroscopy, and the resonance frequency of the film/substrate layered specimen is calculated using the Rayleigh-Ritz method. ${ }^{16}$

In the Rayleigh-Ritz method, because no analytical solution exists for the displacements in a layered-rectangular parallelepiped specimen subjected to free vibration, we approximate 
them using linear combinations of basis functions;

$$
u_{i}\left(x_{1}, x_{2}, x_{3}, t\right)=\sum_{k}^{N} U_{k}^{i} \psi_{k}^{i}\left(x_{1}, x_{2}, x_{3}\right) e^{j \omega t},
$$

where, the $x_{3}$ axis is along the film-thickness direction, and the $x_{1}$ and $x_{2}$ axes lie parallel to the film surface. $\psi$ is the basis function, and $U^{i}$ the expansion coefficient. $\omega$ is the angular frequency. For layered-rectangular parallelepiped specimens, discontinuous displacement gradients arise at the film/substrate interface, from their different elastic constants. For this, the dependence of displacement on the $x_{3}$ direction must be separated from the others as,

$$
\psi_{k}^{i}\left(x_{1}, x_{2}, x_{3}\right)=\zeta_{l}^{i}\left(x_{1}, x_{2}\right) \eta_{m}^{i}\left(x_{3}\right) .
$$

For $\eta_{m}^{i}\left(x_{3}\right)$, one-dimensional Lagrangian interpolation polynomials are used following Heyliger. ${ }^{16}$ The film/substrate specimen is divided into $n_{t}+1$ layers, and the displacements are linearly interpolated within the layers. For $\zeta_{l}^{i}\left(x_{1}, x_{2}\right)$, we use the power series of $x_{1}^{r} x_{2}^{s}(r, s=0,1,2, \ldots)$. It is important to use higher-order basis functions to accurately calculate resonance frequency. In the case of thin films, the number of antinodes of displacements is larger in the in-plane direction than in the out-of-plane direction, because the flexural rigidity of thin films is smaller in the in-plane direction than in the out-of-plane direction. Therefore, the calculation of the resonance frequency of thin films particularly requires the even higherorder basis functions. ${ }^{17}$ We here evaluate the effect of the maximum basis-function orders $n_{t}$ and $n_{p}(=r+s)$ on the calculated resonance frequency of a $\mathrm{Cu}$-fim/Si-substrate layered specimen. The edges of the Si substrate are parallel to $\langle 100\rangle,\langle 010\rangle$, and $\langle 001\rangle$ directions, and the edge lengths are $3.500,3.000$, and $0.100 \mathrm{~mm}$, respectively. The thickness of the $\mathrm{Cu}$ film is $200 \mathrm{~nm}$.

Figure 1 shows the resonance frequencies calculated with various $n_{p}$ values. When $n_{p}$ increases, the calculation accuracy improves, particularly for higher modes. In this study, we measure the resonance frequencies less than $1.0 \mathrm{MHz}$ and we set $n_{p}=18$. $\left(f^{\left(n_{p}=18\right)}-\right.$ $\left.f^{\left(n_{p}=17\right)}\right) / f^{\left(n_{p}=17\right)}$ is then smaller than $0.1 \%$. This calculation error is negligible compared with the measurement errors in dimensions, mass densities and resonance frequencies of the film and substrate. The same evaluation was performed for $n_{t}$. We confirmed that the contribution of $n_{t}$ to resonance frequency is smaller than that of $n_{p}$, and we set $n_{t}=11$. $\left(f^{\left(n_{t}=11\right)}-f^{\left(n_{t}=10\right)}\right) / f^{\left(n_{t}=10\right)}$ is then smaller than $0.02 \%$, which is much smaller than the measurement errors in other parameters.

The volume fraction of the film to the substrate is usually small, and the contribution of the film elastic constants to resonance frequency is less significant compared with the other parameters; as will be described later, the normalized contribution of the in-plane elastic constant $C_{11}$ to the resonance frequency $f,(\Delta f / f) /\left(\Delta C_{11} / C_{11}\right)$, is typically $0.4 \%$ for the $\mathrm{Cu} / \mathrm{Si}$-layer specimens that we used in this study. Therefore, in order to detect the small 
recovery in the thin film $C_{i j}$, resonance frequency has to be measured accurately. Conventional transducers require coupling agents, giving additional mechanical resistance, and they cannot measure resonance frequency in vacuum during the deposition. To solve this problem, we used a homemade tripod transducer set, which consists of two needle-shape piezoelectric transducers and a needle-shaped thermocouple. The set requires no coupling agents, and measurement error of resonance frequency is smaller than $0.01 \%$. The tripod transducer is installed in the sputtering chamber, and resonance frequency is measured continuously before, during and after the deposition. Details of the equipment have been explained in our earlier publication. ${ }^{11}$

A Ag film was deposited on monocrystal Si substrates, measuring $3.5 \times 3.0 \times 0.1 \mathrm{~mm}^{3}$ using magnetron sputtering at room temperature. The background pressure was less than $3.0 \times 10^{-4} \mathrm{~Pa}$, and the Ar pressure was $0.4 \mathrm{~Pa}$ during the deposition. The deposition rate was $1.4 \AA / s$. Two Ag films were deposited, and their film thicknesses were determined to be 128 and $255 \mathrm{~nm}$ by the x-ray reflection method, ${ }^{18,19}$ respectively.

\section{Results}

Figure 2 shows the frequency shift and temperature of the Si substrate measured using the tripod transducer. They were measured every $8 \mathrm{~s}$. The temperature increased during the deposition by $0.7{ }^{\circ} \mathrm{C}$, and gradually decreased after the deposition. The monitored resonance frequency of the $\mathrm{Si}$ substrate was $783.3 \mathrm{kHz}$, and it belongs to the vibration group $\mathrm{B}_{3 \mathrm{~g} .}{ }^{20}$ The out-of-plane displacement distribution, the so-called vibration pattern, at this resonance frequency was measured by laser-Doppler interferometry, ${ }^{21}$ and is shown in the inset of Fig. 2. Bright regions indicate the antinode, and dark regions the node. The displacement was less than $1 \mathrm{~nm}$. We observed that resonance frequency was stable before the deposition, but that it decreased linearly during the deposition. After the deposition, resonance frequency suddenly increased, and then gradually stabilized.

\section{Discussion}

There are three possible reasons for the frequency change after the deposition: (i) the temperature dependence of the $C_{i j}$ of the Si substrate, (ii) the temperature dependence of the $C_{i j}$ of the $\mathrm{Ag}$ film, and (iii) the recovery of the thin film $C_{i j}$. We calculate the temperature effect on the frequency change using the reported temperature dependence of $C_{i j},{ }^{22}$ which is shown by open triangles in Fig. 3. It is much smaller than the observed frequency change (open circles in Fig. 3), and is not a predominant factor for the observed frequency shift. The temperature dependence of the $C_{i j}$ of the $\mathrm{Ag}$ film also affects the frequency changes. However, the volume fraction was less than $2.5 \%$, and its effect is negligible compared with that of the Si substrate. For these reasons, we consider that the observed recovery of resonance frequency originates from the recovery of the elastic constants of the Ag film. 
Among the $C_{i j}$ of the thin film, the resonance frequency of a film/substrate layered specimen is most sensitive to the in-plane elastic constant $C_{11}$ (the longitudinal-wave modulus). We calculate the resonance frequency of the $\mathrm{B}_{3 \mathrm{~g}}$ mode by varying $C_{11}$, and relate the frequency shift to the recovering $C_{11}$. The relationship is shown in Fig. 4 . From this relationship, the normalized contribution of $C_{11}$ to the resonance frequency, $(\Delta f / f) /\left(\Delta C_{11} / C_{11}\right)$, is deduced to be $0.39 \%$, and we found that a $0.062 \%$ increment in resonance frequency corresponds to a $16.0 \%$ increment in $C_{11}$. For a 128 -nm-thick $\mathrm{Ag}$ film, the recovery increased $C_{11}$ by $22 \%$. In this calculation, we assumed that the resonance frequency only depends on the dominant $C_{11}$.

The recovery behavior of resonance frequency is apparently similar to that of residual stress reported by Chason et al. ${ }^{10}$ They explained the recovery of residual stress by the flow of atoms along the grain boundary. During the deposition, the increase in surface chemical potential induces atoms to flow into the grain boundary, creating compressive stress in the film. After the deposition, the surface chemical potential rapidly decreases, and the atoms flow out of the grain boundary to the surface. Then, the time-resolved relaxation of residual stress is reasonably explained by an exponential function, $\exp (-\alpha t)$. Here, $\alpha$ is the recovery rate, and when $\alpha$ is small the recovery progresses slowly. Following their study, we evaluate the recovery rate by fitting the exponential function to the observed recovery of resonance frequency. Because the resonance frequency $f$ is proportional to the square root of the corresponding specific elastic constant $C$, the frequency shift $\Delta f$ is expressed using $\Delta C$ as $\Delta f / f=\Delta C / 2 C$. Therefore, we can evaluate the recovery ratio of $C$ from that of $f$. Figure 3 shows the thus-fitted curve, which agrees well with the experimental result. We obtained recovery rates of 0.019 and 0.012 $\mathrm{s}^{-1}$ for the 128 and $255 \mathrm{~nm}$ films, respectively. These are close to the reported recovery rate of residual stress, $0.0093 \mathrm{~s}^{-1} .10$ The flow of atoms at the grain boundaries, therefore, contributes to the recovery of both the elastic constant and residual stress; during the deposition, the atoms flow into the grain boundary, and after the deposition, excess atoms flow out of the grain boundary, causing a crystalline arrangement at grain boundaries.

\section{Conclusions}

In this study, we observed the recovery of the elastic constants of $\mathrm{Ag}$ films deposited on Si substrates using resonant-ultrasound spectroscopy. The relaxation behavior was similar to the reported one for residual stress, and it was indicated that the flow of atoms at grain boundaries contributes to the recovery of Ag thin films. 


\section{References}

1) N. Nakamura, H. Ogi, T. Ichitsubo, M. Hirao, N. Tatsumi, T. Imai, and H. Nakahata: J. Appl. Phys. 94 (2003) 6405.

2) N. Nakamura, H. Ogi, and M. Hirao: Acta Mater. 52 (2004) 765.

3) H. Ogi, N. Nakamura, H. Tanei, R. Ikeda, M. Hirao, and M. Takemoto: Appl. Phys. Lett. 86 (2005) 231904.

4) H. Tanei, N. Nakamura, H. Ogi, M. Hirao, and R. Ikeda: Phys. Rev. Lett. 100 (2008) 3847.

5) R. Koch and R. Abermann: Thin Solid Films 140 (1986) 217.

6) D. Winau, R. Koch, A. Führmann, and K. H. Rieder: J. Appl. Phys. 70 (1991) 3081.

7) A. L. Shull and F. Spaepen: J. Appl. Phys. 80 (1996) 6243.

8) J. A. Floro, S. J. Hearne, J. A. Hunter, P. Kotula, E. Chason, S. C. Seel, and C. V. Thompson: J. Appl. Phys. 89 (2001) 4886.

9) M. Pletea, W. Brückner, H. Wendrock, J. Thomas, R. Kaltofen, and R. Koch: J. Appl. Phys. 101 (2007) 073511.

10) E. Chason, B. W. Sheldon, L. B. Freund, J. A. Floro, and S. J. Hearne: Phys. Rev. Lett. 88 (2002) 156103.

11) N. Nakamura, H. Ogi, T. Nakashima, M. Hirao, and M. Nishiyama: Jpn. J. Appl. Phys. 46 (2007) 4450.

12) I. Ohno: J. Phys. Earth 24 (1976) 355.

13) A. Migliori, J. L. Sarrao, W. M. Visscher, T. M. Bell, M. Lei, Z. Fisk, and R. G. Leisure: Physica B 183 (1993) 1.

14) N. Nakamura, H. Ogi, H. Nitta, H. Tanei, M. Fujii, T. Yasui, and M. Hirao: Jpn. J. Appl. Phys. 45 (2006) 053511.

15) H. Tanei, N. Nakamura, Y. Kake, H. Ogi, K. Kusakabe, and M. Hirao: Jpn. J. Appl. Phys. 47 (2008) 3847.

16) P. Heyliger: J. Acoust. Soc. Am. 107 (2007) 1235.

17) N. Nakamura, H. Ogi, M. Hirao, T. Fukuhara, K. Shiroki, and N. Imaizumi: Jpn. J. Appl. Phys. 47 (2008) 3851.

18) H. Kiessig: Ann. Phys. (Leipzig) 10 (1931) 769 [in German].

19) L.G.Parratt: Phys. Rev. 95 (1954) 359.

20) E. Mochizuki: J. Phys. Earth 35 (1987) 159.

21) H. Ogi, K. Sato, T. Asada, and M. Hirao: J. Acoust. Soc. Am. 112 (2002) 2553.

22) G. Simmons and H. Wang: Single Crystal Elastic Constants and Calculated Aggregate Properties: A Handbook, (The MIT Press, Cambridge, MA, 1971) 85. 


\section{Figure Captions}

Fig. 1 Dependence of calculated resonance frequency on the order of power series $n_{p}$.

Fig. 2 Observed (a) temperature and (b) resonance frequency of Si substrate before, during, and after the deposition of Ag. The thickness of the deposited film is $255 \mathrm{~nm}$. The monitored resonance frequency of $\mathrm{Si}$ substrate is $783.3 \mathrm{kHz}$. The inset shows a distribution figure of the out-of-plane displacement at the surface of a Si substrate.

Fig. 3 Open circles denote the as-measured resonance frequency shift of $\operatorname{Ag}(255 \mathrm{~nm}) / \operatorname{Si}(0.1$ $\mathrm{mm}$ ) specimen just after the deposition. Open triangles denote the calculated temperature dependence of resonance frequency. The solid line indicates the exponential approximation curve to the as-measured frequency shift.

Fig. 4 Relationship between the elastic constant of the Ag thin film and the frequency shift of the $\mathrm{Ag}(255 \mathrm{~nm}) / \mathrm{Si}(0.1 \mathrm{~mm})$ specimen. 
Jpn. J. Appl. Phys.

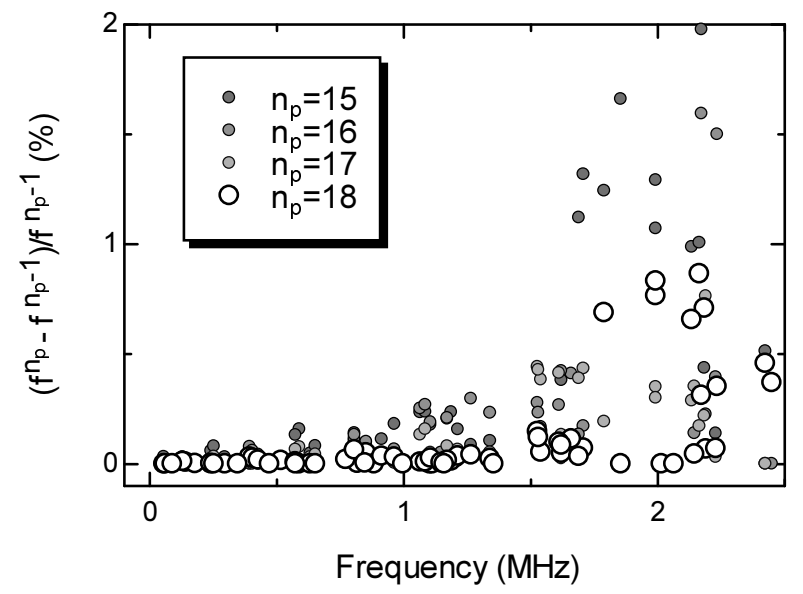

Fig. 1. 
Jpn. J. Appl. Phys.

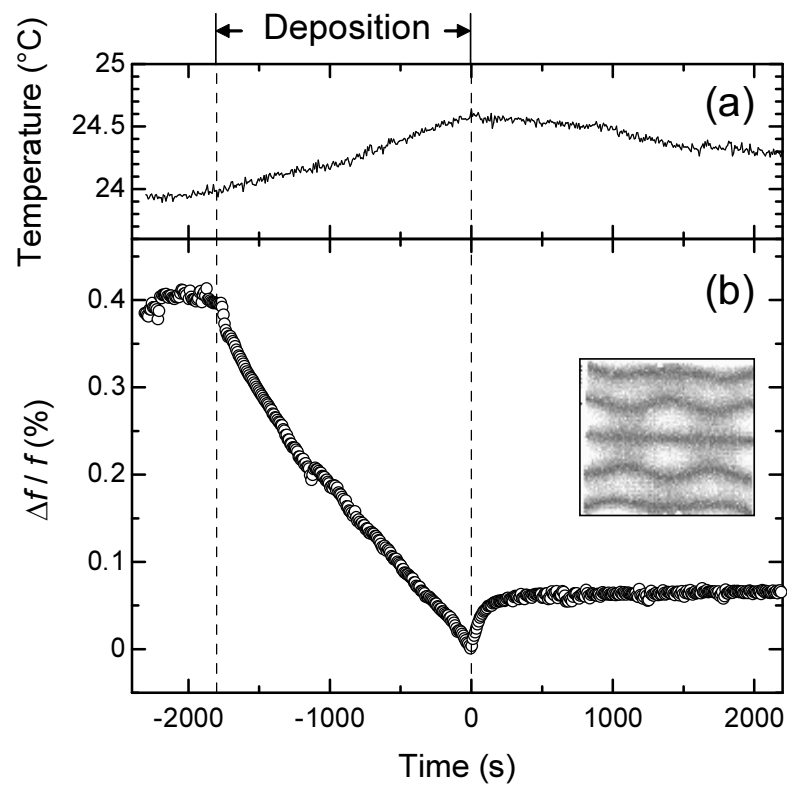

Fig. 2. 
Jpn. J. Appl. Phys.

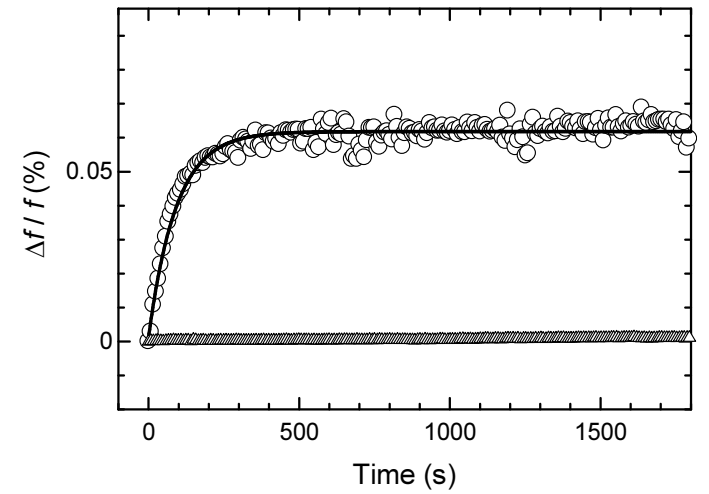

Fig. 3. 
Jpn. J. Appl. Phys.

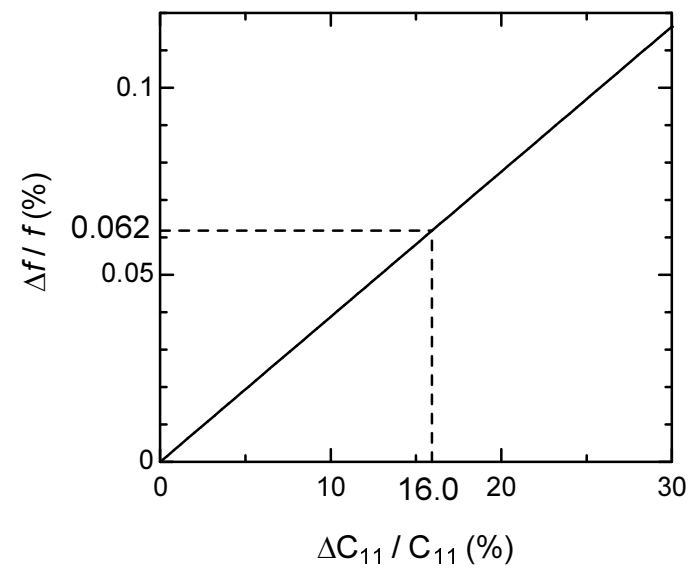

Fig. 4. 